\title{
High Order Asymptotic Expansions of a Good-Bad-Ugly Wave Equation
}

\author{
Miguel Duarte ${ }^{1,2}$, Justin Feng ${ }^{2}$, Edgar Gasperín ${ }^{2,3}$, and David Hilditch ${ }^{2}$ \\ ${ }^{1}$ CAMGSD, Departamento de Matemática, Instituto Superior Técnico IST, \\ Universidade de Lisboa UL, Avenida Rovisco Pais 1, 1049 Lisboa, Portugal, \\ ${ }^{2}$ CENTRA, Departamento de Física, Instituto Superior Técnico IST, \\ Universidade de Lisboa UL, Avenida Rovisco Pais 1, 1049 Lisboa, Portugal, \\ 3 Institut de Mathématiques de Bourgogne (IMB), UMR 5584, CNRS, \\ Université de Bourgogne Franche-Comté, F-21000 Dijon, France
}

\begin{abstract}
A heuristic method to find asymptotic solutions to a system of non-linear wave equations near null infinity is proposed. The non-linearities in this model, dubbed good-bad-ugly, are known to mimic the ones present in the Einstein field equations (EFE) and we expect to be able to exploit this method to derive an asymptotic expansion for the metric in General Relativity (GR) close to null infinity that goes beyond first order as performed by Lindblad and Rodnianski for the leading asymptotics. For the good-bad-ugly model, we derive formal expansions in which terms proportional to the logarithm of the radial coordinate appear at every order in the bad field, from the second order onward in the ugly field but never in the good field. The model is generalized to wave operators built from an asymptotically flat metric and it is shown that it admits polyhomogeneous asymptotic solutions. Finally we define stratified null forms, a generalization of standard null forms, which capture the behavior of different types of field, and demonstrate that the addition of such terms to the original system bears no qualitative influence on the type of asymptotic solutions found.
\end{abstract}

\section{INTRODUCTION}

Although the concept of null infinity has been present in General Relativity (GR) for a long time, dating back at least to the works of Penrose, Newman, Bondi and Sachs - see for instance [1/5, there are still questions to be answered about the structure of spacetime nearby. From the point of view of mathematical relativity, null infinity plays a central role in the resolution of open problems such as the weak cosmic censorship conjecture, the (non) peeling properties and global stability analysis of spacetimes. From an astrophysical perspective, null infinity should also play an important role since gravitational radiation is not localizable and hence it is only well defined at null infinity. The latest achievements in gravitational wave astronomy are coupled to advances in numerical relativity since the former rely on the accurate calculation of waveforms from astrophysically relevant scenarios. However, the waveforms that are routinely computed in numerical relativity codes are evaluated at a large but finite radius and extrapolated to infinity. Despite that the latter has proven to work, from a mathematical point of view these wave forms should thus be computed directly at null infinity. There have been various approaches to include null infinity in the computational domain. For instance, the work of Hübner [6, 7] and Frauendiener [8] makes use of the conformal Einstein field equations (CEFE) introduced by Friedrich [9, 10] building upon Penrose's idea of bringing null infinity to a finite coordinate distance by means of a conformal compactification [1]. Although the CEFE provide a geometric approach to the problem of the inclusion of null infinity, the standard methods of numerical relativity that have proven to work well for the strong field region of spacetimes of physical interest cannot be trivially lifted over. In particular, this approach has not yet been used for compact binary evolutions.

To overcome this situation a variety of approaches have been pursued, including using Cauchy-Characteristic Matching 11 and the use of a suitable hyperboloidal initial value problem. The latter involves providing initial data on a hyperboloidal slice, a spacelike hypersurface that intersects future null infinity. These slices are not Cauchy hypersurfaces, as their domain of dependence does not cover the whole spacetime. The main technical problem with this approach is that it results in formally singular terms which complicates significantly their mathematical analysis as well as their numerical implementation. Although challenging, this type of singular equation has been treated numerically in spherical symmetry [12 15].

In light of the above, a relevant problem to be solved on the analytical side, with direct implications for numerical work, is the construction of an alternative to the CEFEs using more standard formulations of the Einstein field equations (EFE) but insisting on including null infinity. A recent proposal to make inroads into the construction of such a formulation in the hyperboloidal set up is to use a dual frame approach [16], which consists essentially of decoupling coordinates from the tensor basis and carefully choosing each of them. This allows one to write the EFE in generalized harmonic gauge (GHG) and then solve them in hyperboloidal coordinates. Various different aspects of this proposal have been investigated [17 20]. Here we give just a brief overview. An essential prerequisite for this to work is the satisfaction of the coordinate lightspeed condition [18. As discussed in [18, the coordinate lightspeed condition is related to the weak null condition [21]. The former is the requirement that derivatives of the radial coordinate lightspeeds have a certain fall-off near null infinity, while the latter is expected to be a sufficient condition on the non-linearities 
of a quasilinear wave equation for establishing small data global existence. Although it has not been shown in full generality that the weak null condition implies small data global existence, a recent work by Keir 22 proved that if a system of quasilinear wave equations satisfies the $h i$ erarchical weak null condition, then small data global existence is guaranteed. Hörmander's asymptotic system, a heuristic method that predicts the fall-off of solutions to systems of quasi-linear wave equations, was used in 18 to show that through constraint addition, one can guarantee that the resulting field equations satisfy the lightspeed condition beyond the initial data.

The work in 17] and [18] together shows that formally singular terms can be avoided by using the dual foliation formalism [16] in combination with hyperboloidal coordinates and GR in GHG. However, even the simplest choice of variables shows the existence of metric components with a fall-off of the type $O\left(R^{-1} \log R\right)$, with $R$ a suitably defined radial coordinate. This can cause problems in numerical evolutions. In [19, the authors use a toy model composed of wave equations with non-linearities of the same kind as those present in the EFE to show that these logarithmically divergent terms can be explicitly regularized by a non-linear change of variables. This toy model is called the good-bad-ugly model as it splits the evolved fields into three categories according to their fall-off near null infinity, and it is known to satisfy the weak null condition.

In this work we generalize earlier results on the goodbad-ugly model, laying out a heuristic method to predict the type of decay of terms beyond the leading ones in a very large class of systems of non-linear wave equations near null infinity. This provides us with the knowledge of where log-terms may appear in asymptotic expansions so that we are able to manage those terms appropriately in the numerics. The adjective heuristic in this context is used to emphasize that the connection between the original weak null condition introduced by Lindblad and Rodnianski in 21] and small data global existence has not been proven yet, at least not in full generality. To remove this adjective one would need to prove suitable estimates for our formal expansions. This goes beyond the scope of the present article.

In Sections II and III we outline our geometric setup and basic assumptions. In section IV we begin our analysis proper by considering the same model used in [19. We show by induction that the bad field may have logs at every order in $R^{-1}$ and the ugly field may have logs from second order onward under certain conditions, while the good field must have no logs at all. Additionally, we present a recursion relation that gives the coefficient associated with any power of $R^{-1}$ of the evolved fields in terms of the previous order, ultimately in terms of the initial data. In section $\mathrm{V}$ the model is generalized to allow the wave operator to be built from a general asymptotically flat metric whose components are allowed to depend analytically on the evolved fields. An induction proof analogous to the one shown in section [IV] is presented to assert that with such a wave operator, the equations are much more deeply coupled and hence all fields may inherit logs from one another, the main difference being the order at which they are allowed to first appear. In fact, the proof shows that the good-bad-ugly model allows for asymptotic solutions which can be written as polyhomogeneous expansions, loosely speaking inverse powerlaw decay in $R$ but with logarithmic obstructions, close to null infinity. Finally, in section VI we generalize the model even further by adding arbitrary linear combinations of what we call stratified null forms, a generalization of the standard notion that knows about the behavior of the three different types of field, to the original system and showing that these terms do not affect the the proof. Because we keep the metric general, naturally it is not possible to find a final recursion relation for the evolved variables as is done in section [V] but once the exact dependence of the metric on the evolved fields is given, it should be possible to find such a relation. Concluding remarks are given in section VII.

\section{GEOMETRIC SET UP}

Representation of the metric: Latin indices will be used as abstract tensor indices while Greek indices will be used to denote spacetime coordinate indices. We assume the existence of a Lorentzian metric $g_{a b}$ with LeviCivita connection $\nabla$ and introduce the coordinate system $X^{\underline{\alpha}}=\left(T, X^{\underline{i}}\right)$, which we require to be asymptotically Cartesian in a sense clarified below. We raise and lower indices with the spacetime metric $g_{a b}$ exclusively. Let $\partial_{\underline{\alpha}}$ and $d X^{\underline{\alpha}}$ be the corresponding vector and covector bases. The covariant derivative associated to $X^{\underline{\alpha}}$ is $\nabla^{\circ}$ and its Christoffel symbols are defined by,

$$
\Gamma[\stackrel{\circ}{\nabla}]_{a}{ }^{b}{ }_{c}=\left(\stackrel{\circ}{\nabla}_{a} \partial_{\underline{\alpha}}^{b}\right)\left(d X^{\underline{\alpha}}\right)_{c}
$$

Additionally we define shell coordinates $X^{\underline{\alpha}^{\prime}}=$ $\left(T^{\prime}, X^{i^{\prime}}\right)=\left(T, R, \theta^{A}\right)$, where the radial coordinate $R$ is related to $X^{\underline{i}}$ in the usual manner as $R^{2}=\left(X^{\underline{1}}\right)^{2}+$ $\left(X^{2}\right)^{2}+\left(X^{3}\right)^{2}$. Let $\partial_{\alpha^{\prime}}$ and $d X^{\alpha^{\prime}}$ be the corresponding vector and co-vector bases. Shell coordinates have an associated covariant derivative $\dot{\nabla}$ with Christoffel symbols,

$$
\Gamma[\dot{\nabla}]_{b}{ }_{c}^{a}=\left(\dot{\nabla}_{b} \partial_{\underline{\alpha}^{\prime}}^{a}\right)\left(d X^{\underline{\alpha}^{\prime}}\right)_{c}
$$

The transition tensor between the two covariant derivatives is defined by,

$$
\Gamma\left[\stackrel{\circ}{\nabla}^{\circ} \dot{\nabla}\right]_{a}{ }^{b}{ }_{c} v^{c}=\stackrel{\circ}{\nabla}_{a} v^{b}-\dot{\nabla}_{a} v^{b},
$$

where $v^{a}$ is an arbitrary vector field. We define outgoing and incoming null vectors according to,

$$
\begin{aligned}
& \psi^{a}=\partial_{T}^{a}+\mathcal{C}_{+}^{R} \partial_{R}^{a}, \\
& \underline{\psi}^{a}=\partial_{T}^{a}+\mathcal{C}_{-}^{R} \partial_{R}^{a},
\end{aligned}
$$


where $\mathcal{C}_{+}^{R}$ and $\mathcal{C}_{-}^{R}$ are fixed by the requirement that $\psi^{a}$ and $\psi^{a}$ are null vectors with respect to the metric $g_{a b}$. We furthermore define two null co-vectors by,

$$
\sigma_{a}=e^{-\varphi} \psi_{a}, \quad \underline{\sigma}_{a}=e^{-\varphi} \underline{\psi}_{a},
$$

where $\varphi$ is fixed by requiring that

$$
\sigma_{a} \partial_{R}^{a}=-\underline{\sigma}_{a} \partial_{R}^{a}=1
$$

so we can write,

$$
\begin{aligned}
\sigma_{a} & =-\mathcal{C}_{+}^{R} \nabla_{a} T+\nabla_{a} R+\mathcal{C}_{A}^{+} \nabla_{a} \theta^{A}, \\
\underline{\sigma}_{a} & =\mathcal{C}_{-}^{R} \nabla_{a} T-\nabla_{a} R+\mathcal{C}_{A}^{-} \nabla_{a} \theta^{A} .
\end{aligned}
$$

We choose to write the inverse spacetime metric as,

$$
g^{a b}=-2 \tau^{-1} e^{-\varphi} \psi^{(a} \underline{\psi}^{b)}+\phi^{a b},
$$

where the null vectors satisfy,

$$
\begin{aligned}
\sigma_{a} \psi^{a} & =\underline{\sigma}_{a} \underline{\psi}^{a}=0, \\
\sigma_{a} \underline{\psi}^{a} & =\underline{\sigma}_{a} \psi^{a}=-\tau,
\end{aligned}
$$

with $\tau:=\mathcal{C}_{+}^{R}-\mathcal{C}_{-}^{R}$. In (8), the normalization of the first term is carefully chosen so that,

$$
\phi^{a b} \sigma_{b}=\not g^{a b} \underline{\sigma}_{b}=0
$$

and $\not \not^{b}{ }_{a}$ therefore serves as a projection operator orthogonal to these two covectors. Note that $g^{a b}$ is not the inverse induced metric on level sets of $T$ and $R$, as it is not orthogonal to $\nabla_{a} T$ or $\nabla_{a} R$, but rather to $\sigma_{a}$ and $\underline{\sigma}_{a}$. At first sight this seems unsatisfactory geometrically, but since we will be heavily using the method of characteristics it turns out that to be much more convenient to have a simple representation of the vectors $\psi^{a}$ and $\psi^{a}$ than the covectors $\sigma_{a}$ and $\underline{\sigma}_{a}$. Our convention for $\phi^{a b} \overline{\text { follows from }}$ this fact. The metric can be written naturally as,

$$
g_{a b}=-2 \tau^{-1} e^{\varphi} \sigma_{(a} \underline{\sigma}_{b)}+\not g_{a b} .
$$

This way we have ten independent metric functions, namely,

$$
\mathcal{C}_{ \pm}^{R}, \mathcal{C}_{A}^{ \pm}, \varphi, g^{a b}
$$

Tensors projected with $\not_{a}{ }^{b}$ will be denoted adding a slash to the kernel letter $T_{a b} \equiv \not_{a}{ }^{c} \not_{b}{ }^{d} T_{c d}^{\prime}$. The covariant derivative associated to $\oiint_{a b}$ will be denoted as $\not D$, so that,

$$
\not D_{b} v^{a}:=\not_{c}^{a} \not_{b}^{d} \nabla_{d} v^{c} \text {, }
$$

where the vector satisfies $v^{a}=\not g_{b}{ }^{a} v^{b}$. We take the obvious extension for higher rank tensors. Similarly, we define the covariant derivative $\not D$ as,

$$
\stackrel{\circ}{D}_{b} v^{a}:=\not_{c}^{a} \phi_{b}^{d} \stackrel{\circ}{\nabla}_{d} v^{c} \text {. }
$$

We define the vector field $T^{a}:=\partial_{T}^{a}$ and denote the covariant derivative in the direction of $T^{a}$ as $\nabla_{T}$. Analogous notation will be used for directional derivatives along other vector fields. Because we will have to deal with terms proportional to $R^{-n}(\log R)^{m}$, the use of the term 'order' might be confusing, as for the same power of $R^{-1}$ different values of $m$ give rise to different decays. To clarify that, throughout this work, 'order $n$ ' will denote terms proportional to $R^{-n}$.

The good-bad-ugly system: We introduce the following model,

$$
\begin{aligned}
& \stackrel{\circ}{\square}=0, \\
& \square \circ b=\left(\nabla_{T} g\right)^{2}, \\
& \square 0=\frac{2}{R} \nabla_{T} u,
\end{aligned}
$$

where $g, b$ and $u$ stand for good, bad and ugly fields, respectively, $\square$ is called the reduced wave operator and it is defined by $g^{a b} \stackrel{\circ}{\nabla}_{a} \stackrel{\circ}{\nabla}_{b}$. Because we will only be concerned with the large $R$ regime, we are not concerned with regularity at the origin. Therefore, for simplicity, we have adjusted the final equation of the model given in [19] so that the source term appears with a simple coefficient $2 / R$. The metric $g^{a b}$ can be taken to depend on the evolved fields themselves in a manner we will expand upon below. The leading order of the particular case where the metric $g^{a b}$ is the Minkowski metric was studied in detail in 19 .

\section{ASSUMPTIONS}

We need to place certain assumptions on the evolved fields and metric functions that will allow us to formally equate terms of the same order in (15) and retrieve simpler equations that are satisfied, order-by-order, by $g, b$ and $u$ close to null infinity.

Evolved fields: We define a null tetrad $\left\{\psi, \psi, X_{1}, X_{2}\right\}$, where $X_{1}$ and $X_{2}$ are orthogonal to $\psi^{a}$ and $\overline{\psi^{a}}$ and normalized respect to $g_{a b}$, namely, $g_{a b} X_{A}^{a} \psi^{b}=g_{a b} X_{A}^{a} \psi^{b}=0$ and $g_{a b} X_{A}^{a} X_{B}^{b}=\delta_{A B}$, with $A=1,2$. Let $\omega_{g, b}$ be any field in $\{g, b\}$ or any first derivative thereof. Based on insight from 22 we will assume first derivatives of $\omega$ to have the following behavior near null infinity,

$$
\omega_{g, b}=o^{+}\left(R^{-n}\right) \Rightarrow\left\{\begin{array}{l}
\nabla_{\psi} \omega_{g, b}=o^{+}\left(R^{-n-1}\right) \\
\nabla_{\underline{\psi}} \omega_{g, b}=o^{+}\left(R^{-n}\right) \\
\nabla_{X_{A}} \omega_{g, b}=o^{+}\left(R^{-n-1}\right)
\end{array}\right.
$$

with $A \in\{1,2\}$. Here, $f=o^{+}(h)$ as $R \rightarrow \infty$ is defined as the condition,

$$
\exists \epsilon>0: \lim _{R \rightarrow \infty} \frac{f}{h R^{-\epsilon}}=0 .
$$

Note that this condition is a more restrictive version of $f=o(h)$, which can be informally stated as $f$ fallsoff faster than $h^{1+\epsilon}$ as $R$ goes to infinity. In particular, $o^{+}(h)=o\left(h R^{-\epsilon}\right)$. The reason why we make this 
slightly stronger assumption will become apparent once we start integrating error terms in the next section. Derivatives along $\psi^{a}$ and $X_{A}$ are called good derivatives, while the ones along $\psi^{a}$ are called bad derivatives. This naming convention is motivated by the fact that, for fields satisfying equations like ours, the former improve the fall-off of the argument, whereas the latter do not. Let $\omega_{u}$ be the field $u$ or any first derivative thereof. We know from 19 that, in the case that $\square$ is built from the Minkowski metric, derivatives of $u$ have different asymptotics from the other fields, so we assume,

$$
\omega_{u}=o^{+}\left(R^{-n}\right) \Rightarrow\left\{\begin{array}{l}
\nabla_{\psi} \omega_{u}=o^{+}\left(R^{-n-1}\right) \\
\nabla_{\psi} \omega_{u}=o^{+}\left(R^{-n-1}\right) \\
\nabla_{X_{A}} \omega_{u}=o^{+}\left(R^{-n-1}\right)
\end{array} .\right.
$$

We make this set of assumptions using for example $o^{+}\left(R^{-n}\right)$ instead of the more restrictive $O\left(R^{-n-1}\right)$, because previous work has shown that similar equations have asymptotic solutions proportional to, for instance, $R^{-1} \log R$, and naively using big $O$ notation would not permit such solutions [19. Furthermore, we are interested in physically relevant solutions, so we cannot allow fields which do not decay near null infinity. Therefore, we restrict our attention to a space of initial data in which there is decay near null infinity, i.e.,

$$
g=o^{+}(1), \quad b=o^{+}(1), \quad u=o^{+}(1) .
$$

Let $\mathcal{S}$ be a Cauchy surface defined by $T=T_{0}$, where $T_{0}$ is a constant. In order to allow for nonzero ADM mass and linear momentum, we choose initial data which decays at spacelike infinity as,

$$
\begin{aligned}
& \phi=O_{\mathcal{S}}\left(R^{-1}\right), \\
& \nabla_{T} \phi=O_{\mathcal{S}}\left(R^{-2}\right),
\end{aligned}
$$

where the subscript $\mathcal{S}$ is used to say that this fall-off is required on a spatial slice, rather than at null infinity. This is not the most general choice of initial data which allows for nontrivial ADM mass and linear momentum, but it is broad enough to include practically all spacetimes of interest.

Metric functions: As derivatives of our metric functions will, in general, be present in the field equations, we must have a way to collect them in powers of $R^{-1}$. Therefore we require that the metric functions may be written as,

$$
\begin{aligned}
& \mathcal{C}_{ \pm}^{R}= \pm 1+\gamma_{1}^{ \pm}, \\
& \mathcal{C}_{A}^{ \pm}=R \gamma_{2}^{ \pm}, \\
& \varphi=\gamma_{3} \\
& g^{a b}=\not^{a b}+R^{-2} \gamma_{4}^{a b},
\end{aligned}
$$

where $\not^{a b}$ is the inverse metric on the round 2-sphere of radius $R$ and the $\gamma$ 's are analytic functions of only the evolved fields in a neighborhood of null infinity, $\gamma=$ $\gamma(g, b, u)$. Moreover, we are interested in studying spacetimes with metrics that asymptote to the Minkowski metric as we approach $\mathscr{I}^{+}$, i.e. asymptotically flat metrics. This implies that the $\gamma$ functions must go to zero as we approach null infinity, that is,

$$
\left.\gamma(g, b, u)\right|_{\mathscr{I}^{+}}=0 .
$$

\section{FLAT METRIC}

In this section we will study the asymptotics of the good-bad-ugly system 15 with a flat metric $g^{a b}=\eta^{a b}$ near null infinity, where $\eta^{a b}$ is the inverse Minkowski metric. Based on our assumptions on the decay of the fields and their derivatives (16) and (18), we will equate terms of the same order to find simpler equations that will reveal the asymptotics of $g, b$ and $u$. Moreover, this section will serve as a toy model for the next, where we analyze the system for a general asymptotically flat metric. Requiring that the metric be flat implies,

$$
\begin{aligned}
& \mathcal{C}_{ \pm}^{R}= \pm 1, \\
& \varphi=\mathcal{C}_{A}^{ \pm}=0, \\
& g^{a b}=\not^{a b},
\end{aligned}
$$

meaning that all metric components are given, the only unknowns being the evolved fields themselves. The inverse Minkowski metric can then be written in terms of null vectors in the following way,

$$
\eta^{a b}=-\psi^{(a} \underline{\psi}^{b)}+\eta^{a b},
$$

where $\psi^{a}$ and $\underline{\psi}^{a}$ reduce to,

$$
\begin{aligned}
& \psi^{a}=\partial_{T}^{a}+\partial_{R}^{a}, \\
& \underline{\psi}^{a}=\partial_{T}^{a}-\partial_{R}^{a} .
\end{aligned}
$$

The method we want to implement relies on integrating the equations we get along different vector fields. These integrations are made simpler if we work under a Bondilike approach, rewriting the incoming null vector $\psi^{a}$ as a function of the timelike vector $\partial_{T}^{a}$ and the outgoing null vector $\psi^{a}$,

$$
\underline{\psi}^{a}=2 \partial_{T}^{a}-\psi^{a} .
$$

Let $\phi$ be any field in $\{g, b, u\}$. We can expand the wave operator in the following way,

$$
\stackrel{\circ}{\square} \phi=\left(-2 \nabla_{\psi} \nabla_{T}-\frac{2}{R} \nabla_{T}+\frac{2}{R} \nabla_{\psi}+\nabla_{\psi}^{2}+\not \Delta\right) \phi,
$$

where,

$$
\Delta \phi:=\stackrel{\circ}{D}^{a} \stackrel{\circ}{D}_{a} \phi
$$

is the Laplace operator on the 2 -sphere of radius $R$. We treat each equation in (15) separately, and starting with the first. 


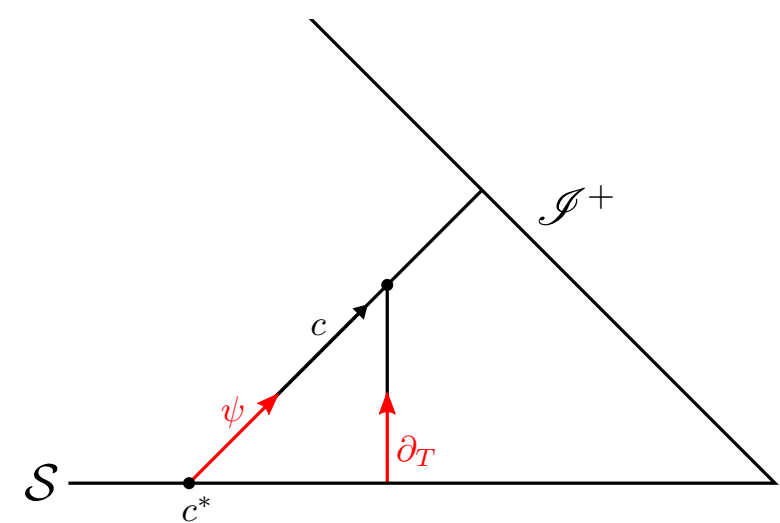

FIG. 1. A schematic of our geometric setup. The method proceeds first by integrating out along $c$, an integral curve of the outgoing null-vector $\psi^{a}$ and then up along integral curves of $\partial_{T}^{a}$.

\section{A. The Good field}

Motivation for induction hypothesis: We begin by rescaling $g$ as,

$$
\mathcal{G}_{1}:=g R,
$$

and plugging that into 27 to get the equation,

$$
-2 \nabla_{\psi} \nabla_{T} \mathcal{G}_{1}+\nabla_{\psi}^{2} \mathcal{G}_{1}+\not \mathcal{G}_{1}=0 .
$$

It is known from 22 that $g$ and its derivatives satisfy (16), so there is a hierarchy among the different terms in (30) which allows us to neglect some of them and end up with a simpler equation that determines $\mathcal{G}_{1}$ to leading order. This is just Hörmander's first order asymptotic system for the wave equation. We know that the second and third terms in (30) are of higher order than the first one because they contain two good derivatives, so the first term must vanish by itself to leading order,

$$
\nabla_{\psi} \nabla_{T} \mathcal{G}_{1}=o^{+}\left(R^{-1}\right)
$$

We want to integrate this expression along integral curves of $\psi^{a}$ and then $\partial_{T}^{a}$. Since integrating error terms is not completely straightforward, we dedicate a paragraph after the present one to proving that error terms remain suitably small under integration. For now we will only pay attention to the leading contributions. Let $c(s)$ be an integral curve of the vector field $\psi^{a}$ that passes through the point $c^{*} \in \mathcal{S}$ at a fiduciary value of $s=s^{*}$ and integrate equation (31) along that curve to get,

$$
\nabla_{T} \mathcal{G}_{1} \simeq \dot{g}_{1}\left(c^{*}\right),
$$

where $\dot{g}_{1}\left(c^{*}\right)=\left.\nabla_{T} g_{1}\right|_{c\left(s^{*}\right)}$. We use $\simeq$ to represent equality at large radius up to error terms that decay faster than those displayed in the expression. For example, we can write $f \simeq R^{-1}$ as short-hand for $f=R^{-1}+o^{+}\left(R^{-1}\right)$.
As we have not specified the curve along which the integration was performed, 32 is valid for any $c$ and so we can write $\left(\psi^{*}\right.$ denoting the dependence on the initial data at $\left.c^{*}\right)$,

$$
\nabla_{T} \mathcal{G}_{1} \simeq \dot{g}_{1}\left(\psi^{*}\right)
$$

where $\dot{g}_{1}\left(\psi^{*}\right)$ is fixed along any particular integral curve of $\psi^{a}$. The exact same method will be used in the rest of this work whenever integrating along integral curves of $\psi^{a}$. Integrating $\sqrt{33}$ in $T$ we get,

$$
\mathcal{G}_{1} \simeq \int_{T_{0}}^{T} \dot{g}_{1}\left(\psi^{*}\right) d T^{\prime}+m_{g, 1},
$$

where $m_{g, 1}$ is a scalar function that is independent of $T$ and we choose it to be independent of $R$ as well. In fact, our choice of initial data 20 requires that all of the $m_{\phi, 1}$ functions throughout the rest of this work are independent of $R$. Moreover, because we impose $(20)$, that choice implies that $\dot{g}_{1}\left(\psi^{*}\right)$ fall-off like $O_{\mathcal{S}}\left(R^{-1}\right)$ because,

$$
\nabla_{T} g \simeq \frac{\dot{g}_{1}\left(\psi^{*}\right)}{R}
$$

Let us now analyze the leading error terms. We define the function $\mathcal{G}_{2}$ as,

$$
\frac{\mathcal{G}_{2}}{R}:=\mathcal{G}_{1}-G_{1}\left(\psi^{*}\right)=o^{+}(1),
$$

where,

$$
G_{1}\left(\psi^{*}\right):=\int_{T_{0}}^{T} \dot{g}_{1}\left(\psi^{*}\right) d T^{\prime}+m_{g, 1},
$$

and assume that it also satisfies $(16)$. Then from $(30)$ we get,

$$
2 \nabla_{\psi}\left(\frac{1}{R} \nabla_{T} \mathcal{G}_{2}\right)+\nabla_{\psi}^{2} \frac{\mathcal{G}_{2}}{R}+\Delta\left(G_{1}+\frac{\mathcal{G}_{2}}{R}\right)=0 .
$$

Here it pays off to introduce the operator,

$$
\tilde{\Delta}:=R^{2} \not
$$

which makes the order in $R^{-1}$ explicit, as $\triangle$ amounts to two good derivatives. Once again, collecting the lowest order terms we get, asymptotically,

$$
2 \nabla_{\psi}\left(\frac{1}{R} \nabla_{T} \mathcal{G}_{2}\right)+\frac{1}{R^{2}} \tilde{X}_{G_{1}}=o^{+}\left(R^{-2}\right) .
$$

which gives,

$$
\mathcal{G}_{2} \simeq-\frac{1}{2} \int_{T_{0}}^{T} \tilde{X}_{G_{1}} d T^{\prime}+R \int_{T_{0}}^{T} \dot{g}_{2}\left(\psi^{*}\right) d T^{\prime}+m_{g, 2} .
$$

In order to integrate along an integral curve of $\psi^{a}$ we parameterize $c$ using the radial coordinate $R$ so that we get,

$$
\int_{c} \frac{1}{R^{n}} d R=-\frac{1}{(n-1) R^{n-1}},
$$


along any $c$. Note that the second term on the RHS of (41) grows like $R$, which would contradict (36). However, if we were to write $\nabla_{T} g$ with what we know already at first order and (41) as it is, we would get,

$$
\begin{aligned}
\nabla_{T} g & =\frac{\nabla_{T} G_{1}}{R}+\frac{\nabla_{T} \mathcal{G}_{2}}{R^{2}} \\
& \simeq \frac{1}{R}\left(\dot{g}_{1}+\dot{g}_{2}\right)+\frac{1}{2} \tilde{\Delta} G_{1},
\end{aligned}
$$

which implies that $\dot{g}_{2}$ can be absorbed into $\dot{g}_{1}$. Therefore we can choose solutions with $\dot{g}_{2}=0$ without any loss of generality, so that,

$$
\mathcal{G}_{2} \simeq G_{2}\left(\psi^{*}\right)
$$

where,

$$
G_{2}\left(\psi^{*}\right):=\frac{1}{2} \int_{T_{0}}^{T} \tilde{\Delta} G_{1} d T^{\prime}+m_{g, 2} .
$$

Equations (34) and (44) suggest that the field $g$ may be written as,

$$
g=\sum_{n=1}^{\infty} \frac{G_{n}\left(\psi^{*}\right)}{R^{n}}
$$

and we prove that result shortly. However it is worth pausing here for a moment to take a closer look at how the error terms behave under integration.

Integration of error terms: What we aim to show here is that if $f=o^{+}\left(R^{-n}\right)$ then,

$$
\int f d R=o^{+}\left(R^{-n+1}\right),
$$

for all $n \in \mathbb{N}$. In other words, we want to show,

$$
\lim _{R \rightarrow \infty} R^{n-1+\epsilon} \int f d R=0 .
$$

Because $\epsilon>0$, and assuming $f$ to be differentiable, we can apply L'Hôpital's rule to the LHS of equation (48) in order to get,

$$
(-n+1-\epsilon)^{-1} \lim _{R \rightarrow \infty} R^{n+\epsilon} f .
$$

By definition we have that $f=o^{+}\left(R^{-n}\right)=o\left(R^{-n-\epsilon}\right)$, which implies directly that (49) is zero. Therefore, (47) must be true. All error terms in this work are of the form $o^{+}\left(R^{-n}\right)$, therefore this result will be used in every integration thereof. Note that if we had made the less restrictive assumption that $f=o\left(R^{-n+1}\right)$ instead of $f=$ $o^{+}\left(R^{-n+1}\right)$, for the case where $n=1$ we would have had to show that,

$$
\lim _{R \rightarrow \infty} \int f d R=0
$$

This would not be possible using the same method and we would not be able to ensure that error terms remain small.
Induction proof: We have seen that,

$$
g=\frac{G_{1}\left(\psi^{*}\right)}{R}+\frac{\mathcal{G}_{2}}{R^{2}}
$$

with $\mathcal{G}_{2}=o^{+}(R)$, so in order to prove our result (46), we only need to show that if $g$ can be written as,

$$
g=\sum_{m=1}^{n-1} \frac{G_{m}\left(\psi^{*}\right)}{R^{m}}+\frac{\mathcal{G}_{n}}{R^{n}}
$$

with $\mathcal{G}_{n}=o^{+}(R)$, then it can be written as,

$$
g=\sum_{m=1}^{n} \frac{G_{m}\left(\psi^{*}\right)}{R^{m}}+\frac{\mathcal{G}_{n+1}}{R^{n+1}},
$$

with $\mathcal{G}_{n+1}=o^{+}(R)$. We assume that each term in $(52)$ satisfies (16), so we have a way to collect terms of the same order. Then we plug $\sqrt{52}$ in $(15)$ and formally equate terms of order $R^{-n-1}$ to find the PDE,

$$
2 R^{n} \nabla_{\psi}\left(\frac{1}{R^{n-1}} \nabla_{T} \mathcal{G}_{n}\right) \simeq[(n-1)(n-2)+\tilde{X}] G_{n-1} .
$$

Integrating equation 54 we find,

$$
\begin{aligned}
\mathcal{G}_{n} \simeq & -\frac{1}{2}\left[n-2+\frac{\tilde{\Delta}}{n-1}\right] \int_{T_{0}}^{T} G_{n-1} d T^{\prime} \\
& +R^{n-1} \int_{T_{0}}^{T} \dot{g}_{n}\left(\psi^{*}\right) d T^{\prime}+m_{g, n} .
\end{aligned}
$$

It can be seen from (55) that, to leading order, $\mathcal{G}_{n}$ does not satisfy (52) for a general $\dot{g}_{n}$. However, $\dot{g}_{n}$ comes with a factor of $R^{n-1}$ so, exactly like in (43), the behavior of those terms is captured by $\dot{g}_{1}$. We can then choose solutions with initial data such that $\dot{g}_{n}=0, \forall n>1$ without loss of generality. Putting aside for a moment the fact that (55) violates $(52)$, we could write the $T$ derivative of $g$ to leading order as,

$$
\nabla_{T} g \simeq \frac{1}{R}\left(\dot{g}_{1}+\ldots+\dot{g}_{n}\right)+\frac{1}{2} \tilde{\AA}_{G_{1}} .
$$

Notice that all the $\dot{g}_{n}$ appear with the same prefactor. So the freedom that the integrations along $\psi$ at each order give us in choosing initial data can in fact be expressed in the choice of one scalar function. This is expected because the fact that we have $n$ differential equations to solve for the different orders in the field $g$ is somewhat artificial, in the sense that they arise from a method to solve a single differential equation. It is therefore natural that once we add all the terms, we are left with only one free function. The same approach will be taken for the fields $b$ and $u$ whenever an integration along integral curves of $\psi^{a}$ is done. This gives the result,

$$
\mathcal{G}_{n} \simeq-\frac{1}{2}\left[n-2+\frac{\tilde{\Delta}}{n-1}\right] \int_{T_{0}}^{T} G_{n-1} d T^{\prime}+m_{g, n}
$$


Clearly, 52 implies (53), so we conclude that $g$ can be written as (46). Moreover, up to $m_{g, n}$, we have a recursion relation that allows us to compute $G_{n}$ from $G_{n-1}$ for any $n>1$,

$$
G_{n}=-\frac{1}{2}\left[n-2+\frac{\tilde{X}}{n-1}\right] \int_{T_{0}}^{T} G_{n-1} d T^{\prime}+m_{g, n} .
$$

\section{B. The Bad field}

Motivation for induction hypothesis: The case of $b$ requires a different hypothesis. Following the same kind of procedure as in the $g$ case, we begin by rescaling $b$ in the following way,

$$
\mathcal{B}_{1}:=b R,
$$

and plugging it into (27). The $b$ equation in 15 can then be written as,

$$
-2 \nabla_{\psi} \nabla_{T} \mathcal{B}_{1}+\nabla_{\psi}^{2} \mathcal{B}_{1}+\not \Delta \mathcal{B}_{1}=R\left(\nabla_{T} g\right)^{2},
$$

where $g$ is now given. The bad field satisfies (16), so collecting terms of the lowest non-trivial order gives the following,

$$
\nabla_{\psi} \nabla_{T} \mathcal{B}_{1} \simeq-\frac{1}{2 R}\left(\nabla_{T} G_{1}\right)^{2}
$$

We can integrate 61 to get,

$\mathcal{B}_{1} \simeq-\frac{1}{2} \log R \int_{T_{0}}^{T}\left(\nabla_{T} G_{1}\right)^{2} d T^{\prime}+\int_{T_{0}}^{T} \dot{b}_{1}\left(\psi^{*}\right) d T^{\prime}+m_{b, 1}$

where $\dot{b}_{1}\left(\psi^{*}\right)$ is a scalar function. The behavior of $b$ to leading order differs from that of $g$ as it has a term that grows with $\log R$. This result is in accordance with 19 . We define the function $\mathcal{B}_{2}$ as,

$$
\frac{\mathcal{B}_{2}}{R}:=\mathcal{B}_{1}-B_{1}=o^{+}(1),
$$

where,

$$
\begin{aligned}
& B_{1}:=B_{1,0}\left(\psi^{*}\right)+B_{1,1}\left(\psi^{*}\right) \log R, \\
& B_{1,0}\left(\psi^{*}\right):=\int_{T_{0}}^{T} \dot{b}_{1}\left(\psi^{*}\right) d T^{\prime}+m_{b, 1}, \\
& B_{1,1}\left(\psi^{*}\right):=-\frac{1}{2} \int_{T_{0}}^{T}\left(\nabla_{T} G_{1}\right)^{2} d T^{\prime},
\end{aligned}
$$

and assume that it also satisfies (16). The subscripts $n$ and $k$ in $B_{n, k}$ stand for the power of $R^{-1}$ and the power of $\log R$ associated with $B_{n, k}$, respectively, and the same notation will be used throughout this work except in the case of $g$ for a flat metric, where it is obvious that the associated field vanishes when $k$ is non-zero. While this notation seems needlessly cumbersome at this point, it will prove useful in the next section, where we find various combinations of powers of $R^{-1}$ and $\log R$. Replacing (63) in 60 and equating lowest order terms gives,

$2 \nabla_{\psi}\left(\frac{1}{R} \nabla_{T} \mathcal{B}_{2}\right)+\frac{1}{R^{2}} B_{1,1}-\not \Delta B_{1} \simeq-\frac{2}{R^{2}} \nabla_{T} G_{1} \nabla_{T} G_{2}$,

which we can integrate to get,

$$
\begin{aligned}
\mathcal{B}_{2} & \simeq \frac{1}{2}(1-\tilde{\Delta}) \int_{T_{0}}^{T} B_{1,1} d T^{\prime}-\frac{1}{2} \tilde{\Delta} \int_{T_{0}}^{T} B_{1} d T^{\prime}+m_{b, 2} \\
& +\int_{T_{0}}^{T} \nabla_{T} G_{1} \nabla_{T} G_{2} d T^{\prime}
\end{aligned}
$$

meaning we can write that,

$$
\mathcal{B}_{2} \simeq B_{2,0}\left(\psi^{*}\right)+B_{2,1}\left(\psi^{*}\right) \log R .
$$

Equations $(62$ and 66 suggest that the field $b$ may be written in the form,

$$
b=\sum_{n=1}^{\infty} \frac{B_{n}}{R^{n}}
$$

with $B_{n}=B_{n, 0}\left(\psi^{*}\right)+B_{n, 1}\left(\psi^{*}\right) \log R$ and we prove this result in the following.

Induction proof: We have computed the bad field to first order,

$$
b=\frac{B_{1}}{R}+\frac{\mathcal{B}_{2}}{R^{2}}
$$

with $\mathcal{B}_{2}=o^{+}(R)$, so in order to prove our result (46), we must show that if $b$ can be written as,

$$
b=\sum_{m=1}^{n-1} \frac{B_{m}}{R^{m}}+\frac{\mathcal{B}_{n}}{R^{n}},
$$

with $\mathcal{B}_{n}=o^{+}(R)$, then it can be written as,

$$
b=\sum_{m=1}^{n} \frac{B_{m}}{R^{m}}+\frac{\mathcal{B}_{n+1}}{R^{n+1}},
$$

with $\mathcal{B}_{n+1}=o^{+}(R)$. We assume all terms in the sum in 69 , as well as $\mathcal{B}_{n}$, satisfy (16) and we plug 69 into (15) to get,

$$
\begin{gathered}
2 R^{n} \nabla_{\psi}\left(\frac{1}{R^{n-1}} \nabla_{T} \mathcal{B}_{n}\right) \simeq-(2 n-3) B_{n-1,1} \\
+[(n-1)(n-2)+\tilde{\Delta}] B_{n-1}-C_{n},
\end{gathered}
$$

where $C_{n}$ is defined as,

$$
C_{n}:=\sum_{i, j=1}^{i+j=n+1} \nabla_{T} G_{i} \nabla_{T} G_{j}
$$


Here, $\sum_{i, j=1}^{i+j=n+1}$ is meant as the sum over terms with any combination of $i$ and $j$ as long as $i, j \geq 1$ and $i+j=n+1$. This can be integrated to get,

$\mathcal{B}_{n} \simeq \frac{1}{2}\left[1-\frac{\tilde{x}}{(n-1)^{2}}\right] \int_{T_{0}}^{T} B_{n-1,1} d T^{\prime}+m_{b, n}$

$-\frac{1}{2}\left[n-2+\frac{\tilde{\Delta}}{n-1}\right] \int_{T_{0}}^{T} B_{n-1} d T^{\prime}+\frac{1}{2(n-1)} \int_{T_{0}}^{T} C_{n} d T^{\prime}$,

as we wanted. As with $\dot{g}_{n}$ above, we choose the functions $\dot{b}_{n}=0, \forall n>1$, effectively absorbing them into $\dot{b}_{1}$ in order to avoid a contradiction with assumptions $(69)$. Finally we get a recursion relation for $B_{n}$,

$B_{n}=\frac{1}{2}\left[1-\frac{\tilde{\Delta}}{(n-1)^{2}}\right] \int_{T_{0}}^{T} B_{n-1,1} d T^{\prime}+m_{b, n}$

$-\frac{1}{2}\left[n-2+\frac{\tilde{\Delta}}{n-1}\right] \int_{T_{0}}^{T} B_{n-1} d T^{\prime}+\frac{1}{2(n-1)} \int_{T_{0}}^{T} C_{n} d T^{\prime}$.

This shows our hypothesis 67), with,

$B_{n, 0}=\frac{1}{2}\left[1-\frac{\tilde{\Delta}}{(n-1)^{2}}\right] \int_{T_{0}}^{T} B_{n-1,1} d T^{\prime}+m_{b, n}$

$-\frac{1}{2}\left[n-2+\frac{\tilde{\Delta}}{n-1}\right] \int_{T_{0}}^{T} B_{n-1,0} d T^{\prime}+\frac{1}{2(n-1)} \int_{T_{0}}^{T} C_{n} d T^{\prime}$.

and,

$$
B_{n, 1}=-\frac{1}{2}\left[n-2+\frac{\tilde{\Delta}}{n-1}\right] \int_{T_{0}}^{T} B_{n-1,1} d T^{\prime},
$$

A closer look at 74 reveals that the only $\log R$ term comes from $B_{n-1}$, and hence it is inherited by all orders from $B_{1}$.

\section{The Ugly field}

Motivation for induction hypothesis: Once again we rescale the field $u$ by $R$ as,

$$
\mathcal{U}_{1}=u R
$$

and plug it into (27). The $u$ equation in 15 can then be written as,

$$
-\frac{2}{R} \nabla_{\psi}\left(R \nabla_{T} \mathcal{U}_{1}\right)+\nabla_{\psi}^{2} \mathcal{U}_{1}+\not \mathcal{U}_{1}=0
$$

The ugly field has a somewhat different behavior from the other two. As can be seen in [19], both good and bad derivatives improve the fall-off of $u$, as opposed to the cases of $g$ and $b$, where only good derivatives improve. This means that all terms in (78) contribute to leading order and one would have to solve the whole equation at once. For this reason, we will focus on solutions that can be decomposed as,

$$
\mathcal{U}_{1}=m_{u, 1}+\frac{\mathcal{U}_{2}}{R}
$$

where $m_{u, 1}$ is independent of $T$ and $R$ and $\mathcal{U}_{2}=o^{+}(R)$. Plugging this into (78) we get,

$$
-\frac{2}{R} \nabla_{\psi} \nabla_{T} \mathcal{U}_{2}+\left(\nabla_{\psi}^{2}+\not \Delta\right)\left(m_{u, 1}+\frac{\mathcal{U}_{2}}{R}\right)=0 .
$$

If we assume $\mathcal{U}_{2}$ to satisfy 16 ,

$$
\begin{aligned}
& 2 \nabla_{\psi} \nabla_{T} \mathcal{U}_{2} \simeq \frac{1}{R} \tilde{\Delta} m_{u, 1} \Rightarrow \\
& \mathcal{U}_{2} \simeq \int_{T_{0}}^{T}\left[\log R \tilde{X} m_{u, 1}+\dot{u}_{2}\left(\psi^{*}\right)\right] d T^{\prime}+m_{u, 2}
\end{aligned}
$$

Equation 81 suggests that the ugly field can be written as,

$$
u=\frac{m_{u, 1}}{R}+\sum_{n=2}^{\infty} \frac{U_{n}}{R^{n}}
$$

with $U_{n}=U_{n, 0}\left(\psi^{*}\right)+U_{n, 1}\left(\psi^{*}\right) \log R$ and we show this result in the following.

Induction proof: As was seen above, the first order term of the ugly field behaves differently from the rest, in that all of its derivatives improve. For this reason we begin the induction proof in the second order term, which has been computed in $(79)$ and 81 . To prove 82 we have to show that if,

$$
u=\frac{m_{u, 1}}{R}+\sum_{m=2}^{n-1} \frac{U_{m}}{R^{m}}+\frac{\mathcal{U}_{n}}{R^{n}},
$$

with $\mathcal{U}_{n}=o^{+}(R)$, then,

$$
u=\frac{m_{u, 1}}{R}+\sum_{m=2}^{n} \frac{U_{m}}{R^{m}}+\frac{\mathcal{U}_{n+1}}{R^{n+1}},
$$

with $\mathcal{U}_{n+1}=o^{+}(R)$. We assume that all orders in $(83)$ and $\mathcal{U}_{n}$ satisfy (16) and we substitute that in (15) to get,

$$
\begin{array}{r}
2 R^{n-1} \nabla_{\psi}\left(\frac{1}{R^{n-2}} \nabla_{T} \mathcal{U}_{n}\right)=-(2 n-3) U_{n-1,1} \\
+[(n-1)(n-2)+\tilde{X}] U_{n-1},
\end{array}
$$

which we can integrate to get, $\forall n>2$,

$$
\begin{aligned}
\mathcal{U}_{n} \simeq & \frac{1}{2}\left[1-\frac{\tilde{\Delta}}{(n-1)^{2}}\right] \int_{T_{0}}^{T} U_{n-1,1} d T^{\prime}+m_{u, n} \\
& -\frac{1}{2}\left[n-2+\frac{\tilde{\Delta}}{n-1}\right] \int_{T_{0}}^{T} U_{n-1} d T^{\prime} .
\end{aligned}
$$


As in the $g$ and $b$ cases, we consider the initial data arising from the $\psi^{a}$ integration $\dot{u}_{n}$ to be zero for all $n>2$. This concludes the proof by induction and we get a final recursion relation for $U_{n}$ in terms of $U_{n-1}$,

$$
\begin{aligned}
U_{n}= & \frac{1}{2}\left[1-\frac{\tilde{\Delta}}{(n-1)^{2}}\right] \int_{T_{0}}^{T} U_{n-1,1} d T^{\prime}+m_{u, n} \\
& -\frac{1}{2}\left[n-2+\frac{\tilde{\Delta}}{n-1}\right] \int_{T_{0}}^{T} U_{n-1} d T^{\prime} .
\end{aligned}
$$

Our results are summarized by the following:

Theorem 1. Let $X^{\underline{\alpha}}=\left(T, X^{\underline{i}}\right)$ be an asymptotically Cartesian coordinate system with an associated covariant derivative $\stackrel{\circ}{\nabla}$. The good-bad-ugly system defined as,

$$
\begin{aligned}
& \stackrel{\circ}{\square} g=0, \\
& \stackrel{\circ}{\square} b=\left(\nabla_{T} g\right)^{2}, \\
& \stackrel{\circ}{\square} u=\frac{2}{R} \nabla_{T} u,
\end{aligned}
$$

where $\stackrel{\circ}{\square}:=\eta^{a b} \stackrel{\circ}{\nabla}_{a} \stackrel{\circ}{\nabla}_{b}$ and $\eta$ is the Minkowski metric, admits formal polyhomogeneous asymptotic solutions near null infinity of the type,

$$
\begin{aligned}
& g=\sum_{n=1}^{\infty} \frac{G_{n}\left(\psi^{*}\right)}{R^{n}}, \\
& b=\sum_{n=1}^{\infty} \frac{B_{n}}{R^{n}}, \\
& u=\frac{m_{u, 1}}{R}+\sum_{n=2}^{\infty} \frac{U_{n}}{R^{n}},
\end{aligned}
$$

where $B_{n}=B_{n, 0}\left(\psi^{*}\right)+B_{n, 1}\left(\psi^{*}\right) \log R$ and $U_{n}=$ $U_{n, 0}\left(\psi^{*}\right)+U_{n, 1}\left(\psi^{*}\right) \log R$ and with initial data on $\mathcal{S}$ of the type,

$$
\left\{\begin{array}{l}
\left.g\right|_{\mathcal{S}}=\sum_{n=1}^{\infty} \frac{m_{g, n}}{R^{n}} \\
\left.b\right|_{\mathcal{S}}=\sum_{n=1}^{\infty} \frac{m_{b, n}}{R^{n}} \\
\left.u\right|_{\mathcal{S}}=\sum_{n=1}^{\infty} \frac{m_{u, n}}{R^{n}}
\end{array} \quad,\left\{\begin{array}{l}
\left.\nabla_{T} g\right|_{\mathcal{S}}=O_{\mathcal{S}}\left(R^{-2}\right) \\
\left.\nabla_{T} b\right|_{\mathcal{S}}=O_{\mathcal{S}}\left(R^{-2}\right) \\
\left.\nabla_{T} u\right|_{\mathcal{S}}=O_{\mathcal{S}}\left(R^{-2}\right)
\end{array}\right.\right.
$$

where $m_{\phi, n}$ are scalar functions that are independent of $T$ and $R$. This is valid outside a compact ball centered at $R=0$. Additionally, the functions $G_{n}$ are given by (37) and (58), $B_{n}$ are given by (64) and (74) and $U_{n}$ by (81) and (87).

Remark 1. Looking at equation (87) we see that the only way for $U_{n}$ to have a term with $\log R$ in it is if $U_{n-1}$ does too. As this is valid for any $n>2$, the orders of $U_{n}$ higher than $U_{2}$ can only have a $\log R$ term if $U_{2}$ does as well. In other words, if we require $\Delta m_{u, 1}=0$, the field $u$ will have no $\log R$ terms at any order. In fact, with that requirement, it can easily be seen that $U_{n}$ satisfies a hypothesis analogous to that of the field $g$, namely,

$$
u=\sum_{n=1}^{\infty} \frac{U_{n}\left(\psi^{*}\right)}{R^{n}}
$$

Remark 2. The good-bad-ugly system 15 admits a static solution that is obtained with the following initial data in $\mathcal{S}$,

$$
\left\{\begin{array}{l}
\left.g\right|_{\mathcal{S}}=\frac{m_{g, 1}}{R} \\
\left.b\right|_{\mathcal{S}}=\frac{m_{b, 1}}{R} \\
\left.u\right|_{\mathcal{S}}=\frac{m_{u, 1}}{R}
\end{array}\right.
$$

where the functions $m_{\phi, 1}$ satisfy the condition $\Delta m_{\phi, 1}=0$. In that case, looking at the recursion relations (58), (74) and (81), we see that the series is truncated at $n=1$ and hence all the higher order terms vanish.

\section{ASYMPTOTICALLY FLAT METRICS}

In this section we follow the same procedure as in the prequel, but this time employing a more general metric whose functions are allowed to depend analytically on the fields $g, b$ and $u$. Since we want to maintain the generality of those functions, the final recursion relation for the good-bad-ugly system will have to be written as a function of $g, b$ and $u$. Nevertheless, we will see that an induction proof analogous to that of the flat metric case can be made for a general asymptotically flat metric under the assumptions given in section III. An asymptotically flat metric that is simply given, rather than occurring as a function of our unknown fields, could be treated similarly.

Expansion of the reduced wave operator: Let $\phi$ be any field in $\{g, b, u\}$ and use (8) to expand the LHS of (15),

$$
\begin{aligned}
\stackrel{\circ}{\square} \phi= & {\left[-\frac{2 e^{-\varphi}}{\tau} \nabla_{\psi} \nabla_{\underline{\psi}}+\frac{2 e^{-\varphi}}{\tau}\left(\stackrel{\circ}{\nabla}_{\psi} \underline{\psi}\right)^{a} \nabla_{a}-\frac{1}{\tau} \stackrel{\circ}{D}^{a} \sigma_{a} \nabla_{\underline{\psi}}\right.} \\
& \left.-\frac{1}{\tau} \not \stackrel{\circ}{D}^{a} \underline{\sigma}_{a} \nabla_{\psi}+\not \Delta\right] \phi .
\end{aligned}
$$

We want to write expression (93) in terms of derivatives of metric functions and $\phi$ along the vector fields $\psi^{a}$ and $\partial_{T}^{a}$. For clarity, let us treat each term individually and put everything together in the end. Using (4), the first term on the RHS turns into,

$$
\nabla_{\psi} \nabla_{\underline{\psi}} \phi=\nabla_{\psi}\left(\frac{\tau}{\mathcal{C}_{+}^{R}} \nabla_{T} \phi+\frac{\mathcal{C}_{-}^{R}}{\mathcal{C}_{+}^{R}} \nabla_{\psi} \phi\right) .
$$

From the second term on the RHS we get,

$$
\left(\stackrel{\circ}{\nabla}_{\psi} \underline{\psi}\right)^{a} \nabla_{a} \phi=\left(\dot{\nabla}_{\psi} \underline{\psi}\right)^{a} \nabla_{a} \phi=\frac{1}{\mathcal{C}_{+}^{R}} \nabla_{\psi} \mathcal{C}_{-}^{R}\left(\nabla_{\psi} \phi-\nabla_{T} \phi\right),
$$

where the first equality comes from the fact that $\Gamma[\stackrel{\circ}{\nabla}, \dot{\nabla}]_{\psi}{ }^{a} \underline{\psi}=0$. The third term can be expanded as,

$$
\begin{aligned}
& \stackrel{\circ}{D}^{a} \sigma_{a} \nabla_{\underline{\psi}} \phi=\phi^{a b}\left(\dot{\nabla}_{a} \sigma_{b}-\Gamma[\stackrel{\circ}{\nabla}, \dot{\nabla}]_{a}{ }^{\sigma}{ }_{b}\right) \nabla_{\underline{\psi}} \phi \\
& =g^{a b}\left(-\nabla_{b} T \nabla_{a} \mathcal{C}^{R}+\nabla_{b} \theta^{A} \nabla_{a} \mathcal{C}_{A}^{+}-\Gamma[\dot{\nabla}, \dot{\nabla}]_{a}{ }^{\sigma}{ }_{b}\right) \nabla_{\underline{\psi}} \phi \\
& =\left(-\frac{\mathcal{C}_{A}}{\tau} \not D^{A} \mathcal{C}^{R}+\not D^{A} \mathcal{C}_{A}^{+}-\phi^{a b} \Gamma[\stackrel{\circ}{\nabla}, \dot{\nabla}]_{a}{ }^{\sigma} b\right) \nabla_{\underline{\psi}} \phi,
\end{aligned}
$$


where $\mathcal{C}_{A}:=\mathcal{C}_{A}^{+}+\mathcal{C}_{A}^{-}$and $\nabla_{\psi} \phi$ should be written in terms of $\nabla_{T} \phi$ and $\nabla_{\psi} \phi$ with (4), whereas the fourth term reads,

$\stackrel{\circ}{D}^{a} \underline{\sigma}_{a} \nabla_{\psi} \phi=\left(-\frac{\mathcal{C}_{A}}{\tau} \not D^{A} \mathcal{C}^{R}+\not D^{A} \mathcal{C}_{A}^{-}-\not g^{a b} \Gamma[\stackrel{\circ}{\nabla}, \dot{\nabla}]_{a}{ }^{\underline{\sigma}}{ }_{b}\right) \nabla_{\psi} \phi$.

Putting all of this together in (93) gives,

$$
\begin{aligned}
\mathcal{C}_{+}^{R} \stackrel{\circ}{\square} \phi=^{-} & \left.-2 e^{-\varphi} \nabla_{\psi} \nabla_{T} \phi+\nabla_{T} \phi(\not)^{a b} \Gamma\left[\nabla^{\circ}, \dot{\nabla}\right]_{a}{ }^{\sigma}{ }_{b}+X_{T}\right) \\
& +\nabla_{\psi} \phi X_{\psi}-\frac{e^{-\varphi} \mathcal{C}_{-}^{R}}{\tau} \nabla_{\psi}^{2} \phi+\mathcal{C}_{+}^{R} \not \Delta \phi
\end{aligned}
$$

where $X_{T}$ and $X_{\psi}$ are,

$$
\begin{aligned}
& \tau X_{T}:=\mathcal{C}_{A} \not D^{A} \mathcal{C}_{-}^{R}-\tau \not D^{A} \mathcal{C}_{A}^{+}+\frac{2 e^{-\varphi} \mathcal{C}_{-}^{R}}{\mathcal{C}_{+}^{R}} \nabla_{\psi} \mathcal{C}_{+}^{R} \\
& \tau X_{\psi}:=\frac{\mathcal{C}_{A}}{\tau} \not D^{A}\left(\mathcal{C}_{-}^{R} \mathcal{C}_{+}^{R}\right)-\mathcal{C}_{-}^{R} \not D^{A} \mathcal{C}_{A}^{+}-\mathcal{C}_{+}^{R} \not D^{A} \mathcal{C}_{A}^{-} \\
& \quad+\mathcal{C}_{-}^{R} \phi^{a b} \Gamma[\stackrel{\circ}{\nabla}, \dot{\nabla}]_{a}{ }^{\sigma}{ }_{b}+\mathcal{C}_{+}^{R} \phi^{a b} \Gamma[\stackrel{\circ}{\nabla}, \dot{\nabla}]_{a}{ }^{\underline{\sigma}}{ }_{b}+\frac{2 e^{-\varphi} \mathcal{C}_{-}^{R}}{\mathcal{C}_{+}^{R}} \nabla_{\psi} \mathcal{C}_{+}^{R}
\end{aligned}
$$

Motivation for induction hypothesis: As the $\gamma$ functions (see (21) ) are analytic functions of the evolved fields at null infinity, we can Taylor expand them around $g=$ $b=u=0$, because the fields are assumed to have decay near null infinity. That gives,

$$
\begin{aligned}
\gamma(g, b, u) & =\left.\sum_{i=0}^{\infty} \sum_{j=0}^{\infty} \sum_{k=0}^{\infty} \frac{g^{i} b^{j} u^{k}}{i ! j ! k !}\left(\frac{\partial^{i+j+k} \gamma}{\partial g^{i} \partial b^{j} \partial u^{k}}\right)\right|_{\mathscr{I}+} \\
& =\left.\frac{\partial \gamma}{\partial g}\right|_{\mathscr{I}+} g+\left.\frac{\partial \gamma}{\partial b}\right|_{\mathscr{I}+} b+\left.\frac{\partial \gamma}{\partial u}\right|_{\mathscr{I}+} u+\ldots,
\end{aligned}
$$

where the second equality uses the fact that $\left.\gamma\right|_{\mathscr{I}^{+}}=0$, because the metric is asymptotically flat. Equation (98) then implies that $\gamma=o^{+}(1)$ and,

$$
\omega_{\gamma}=o^{+}\left(R^{-n}\right) \Rightarrow\left\{\begin{array}{l}
\nabla_{\psi} \omega_{\gamma}=o^{+}\left(R^{-n-1}\right) \\
\nabla_{X_{A}} \omega_{\gamma}=o^{+}\left(R^{-n-1}\right)
\end{array},\right.
$$

where $\omega_{\gamma}$ is any $\gamma$ function or any derivative of it. Note that we intentionally left out any bad derivatives because in order to know the asymptotic behavior of those we would have to specify the dependence of $\gamma$ on $g, b$ and $u$. Remarkably, one can easily check that the expanded form of the reduced wave operator (96) does not include any bad derivatives of metric functions. Let us rescale $g, b$ and $u$ as (29), 59) and (79), respectively. With (99) one can count the order of each term in (96) and see that only the first two terms contribute to leading order. These are exactly the same terms that contribute to leading order in the flat metric case, which means that none of the extra terms that arise from allowing the spacetime to have curvature can possibly contribute to first order. We get the equations,

$$
\begin{aligned}
& \nabla_{\psi} \nabla_{T} \mathcal{G}_{1} \simeq 0 \\
& \nabla_{\psi} \nabla_{T} \mathcal{B}_{1} \simeq-\frac{2}{R}\left(\nabla_{T} G\right)^{2}, \\
& 2 \nabla_{\psi} \nabla_{T} \mathcal{U}_{2} \simeq \frac{1}{R} \tilde{\Delta} m_{u, 1} .
\end{aligned}
$$

Note that, to leading order, $\tilde{X}$ is the Laplacian on the 2 -sphere of unit radius,

$$
\tilde{X}_{\phi} \simeq R^{2} \not \eta^{a b} \stackrel{\circ}{\nabla}_{a}\left(\not^{c}{ }_{b} \stackrel{\circ}{\nabla}_{c} \phi\right)
$$

because $\not^{a b}$ approaches the inverse metric on the 2-sphere of radius $R$. Therefore we have,

$$
\begin{aligned}
& \mathcal{G}_{1} \simeq G_{1,0}\left(\psi^{*}\right) \\
& \mathcal{B}_{1} \simeq B_{1,0}\left(\psi^{*}\right)+B_{1,1}\left(\psi^{*}\right) \log R \\
& \mathcal{U}_{2} \simeq \int_{T_{0}}^{T}\left[\log R \tilde{\not} m_{u, 1}+\dot{u}_{2}\left(\psi^{*}\right)\right] d T^{\prime}+m_{u, 2}\left(T_{0}\right) .
\end{aligned}
$$

In 102 we kept $\mathcal{U}_{2}$ as a function of $m_{u, 1}$ because there is a remark to be made about it at the end of this section. As the metric functions are free to depend upon the evolved fields, the second order equations (third order in the case of $u$ ) may be coupled to first order terms. This means that $\mathcal{G}_{1}-G_{1,0}$, for instance, could have a term proportional to $\log R$ that is coming from $\mathcal{B}_{1}$. On the other hand, non-linearities could give rise to terms proportional to higher powers of $\log R$. This seems to suggest that $g, b$ and $u$ are polyhomogeneous functions where each term can have up to $n$ powers of $\log R$ in the $b$ case, and up to $n-1$ in the $g$ and $u$ cases. Formally, we therefore conjecture

$$
\begin{aligned}
& g=\sum_{n=1}^{\infty} \sum_{k=0}^{n-1} \frac{(\log R)^{k} G_{n, k}\left(\psi^{*}\right)}{R^{n}} \\
& b=\sum_{n=1}^{\infty} \sum_{k=0}^{n} \frac{(\log R)^{k} B_{n, k}\left(\psi^{*}\right)}{R^{n}} \\
& u=\frac{m_{u, 1}}{R}+\sum_{n=2}^{\infty} \sum_{k=0}^{n-1} \frac{(\log R)^{k} U_{n, k}\left(\psi^{*}\right)}{R^{n}} .
\end{aligned}
$$

We proceed by induction as in the the previous cases. From 102 we can already know that to first order in $g$ and $u, \log R$ terms are not allowed, and the conjecture 103 incorporates this property by construction. Truncating at $n=1$, we have seen

$$
\begin{aligned}
& g=\frac{G_{1,0}\left(\psi^{*}\right)}{R}+\frac{\mathcal{G}_{2}}{R^{2}}, \\
& b=\frac{B_{1,0}\left(\psi^{*}\right)+B_{1,1}\left(\psi^{*}\right) \log R}{R}+\frac{\mathcal{B}_{2}}{R^{2}}, \\
& u=\frac{m_{u, 1}}{R}+\frac{U_{2,0}\left(\psi^{*}\right)+U_{2,1}\left(\psi^{*}\right) \log R}{R^{2}}+\frac{\mathcal{U}_{3}}{R^{3}},
\end{aligned}
$$

with $\mathcal{G}_{2}=o^{+}(R), \mathcal{B}_{2}=o^{+}(R)$ and $\mathcal{U}_{3}=o^{+}(R)$, so in order to show 103 , we have to show that if we can write 
the evolved fields as,

$$
\begin{aligned}
g & =\sum_{m=1}^{n-1} \sum_{k=0}^{m-1} \frac{(\log R)^{k} G_{m, k}\left(\psi^{*}\right)}{R^{m}}+\frac{\mathcal{G}_{n}}{R^{n}} \\
b & =\sum_{m=1}^{n-1} \sum_{k=0}^{m} \frac{(\log R)^{k} B_{m, k}\left(\psi^{*}\right)}{R^{m}}+\frac{\mathcal{B}_{n}}{R^{n}} \\
u & =\frac{m_{u, 1}}{R}+\sum_{m=2}^{n-1} \sum_{k=0}^{m-1} \frac{(\log R)^{k} U_{m, k}\left(\psi^{*}\right)}{R^{m}}+\frac{\mathcal{U}_{n}}{R^{n}},
\end{aligned}
$$

where $\mathcal{G}_{n}=o^{+}(R), \mathcal{B}_{n}=o^{+}(R)$ and $\mathcal{U}_{n}=o^{+}(R)$, then we can also write them as,

$$
\begin{aligned}
& g=\sum_{m=1}^{n} \sum_{k=0}^{m-1} \frac{(\log R)^{k} G_{m, k}\left(\psi^{*}\right)}{R^{m}}+\frac{\mathcal{G}_{n+1}}{R^{n+1}} \\
& b=\sum_{m=1}^{n} \sum_{k=0}^{m} \frac{(\log R)^{k} B_{m, k}\left(\psi^{*}\right)}{R^{m}}+\frac{\mathcal{B}_{n+1}}{R^{n+1}} \\
& u=\frac{m_{u, 1}}{R}+\sum_{m=2}^{n} \sum_{k=0}^{m-1} \frac{(\log R)^{k} U_{m, k}\left(\psi^{*}\right)}{R^{m}}+\frac{\mathcal{U}_{n+1}}{R^{n+1}},
\end{aligned}
$$

where $\mathcal{G}_{n+1}=o^{+}(R), \mathcal{B}_{n+1}=o^{+}(R)$ and $\mathcal{U}_{n+1}=o^{+}(R)$. To do this, we must first find what the metric functions, and hence the $\gamma$ functions, behave like if we assume (105).

Behavior of $\gamma$ functions: Let $\phi_{1}$ and $\phi_{2}$ be any of the fields in $\{g, b, u\}$. According to our assumption (105), $\phi_{i}$ with $i \in\{1,2\}$ can be written as

$$
\phi_{i}=\sum_{m=1}^{n-1} \sum_{k=0}^{m} \frac{(\log R)^{k} \Phi_{m, k}\left(\psi^{*}\right)}{R^{m}}+\frac{\Phi}{R^{n}},
$$

for suitable scalar functions $\Phi_{m, k}\left(\psi^{*}\right)$ and $\Phi=o^{+}(R)$. It is then straightforward to check that the product of any two evolved fields can also be written as,

$$
\phi_{1} \phi_{2}=\sum_{m=1}^{n-1} \sum_{k=0}^{m} \frac{(\log R)^{k} \bar{\Phi}_{m, k}\left(\psi^{*}\right)}{R^{m}}+\frac{\bar{\Phi}}{R^{n}},
$$

once again for suitable functions $\bar{\Phi}_{m, k}\left(\psi^{*}\right)$ and $\bar{\Phi}=$ $o^{+}(R)$, which is formally the same as (107). This means that no matter how many times we multiply any powers of the evolved fields, it is always possible to write the resulting product as 108 . If we plug $(105)$ into $\sqrt{98}$ we get in each term a product of powers of the fields $g, b$ and $u$, so we can write any $\gamma$ function as,

$$
\gamma=\sum_{m=1}^{n-1} \sum_{k=0}^{m} \frac{(\log R)^{k} \Gamma_{m, k}\left(\psi^{*}\right)}{R^{m}}+\frac{\Gamma}{R^{n}},
$$

with $\Gamma=o^{+}(R)$.

Induction proof: We plug 105 and 109 into (96) and collect terms proportional to $R^{-n-1}$. In the $g$ equation, for instance, we see that the only terms in (96) that may contain $\mathcal{G}_{n}$ are the first two, while none of the remaining terms may contain $\mathcal{B}_{n}$ or $\mathcal{U}_{n}$. Putting all terms with $\mathcal{G}_{n}, \mathcal{B}_{n}$ and $\mathcal{U}_{n}$ on the LHS and all the rest on the RHS we get the system,

$$
\begin{aligned}
& R^{n} \nabla_{\psi}\left(\frac{1}{R^{n-1}} \nabla_{T} \mathcal{G}_{n}\right) \simeq \Omega_{n-1}^{g}, \\
& R^{n} \nabla_{\psi}\left(\frac{1}{R^{n-1}} \nabla_{T} \mathcal{B}_{n}\right)+\nabla_{T} G_{n} \nabla_{T} G_{1} \simeq \Omega_{n-1}^{b}, \\
& R^{n-1} \nabla_{\psi}\left(\frac{1}{R^{n-2}} \nabla_{T} \mathcal{U}_{n}\right) \simeq \Omega_{n-1}^{u},
\end{aligned}
$$

where on the right hand sides $\Omega_{n-1}^{\phi}$ depend on the functions $\left\{G_{m, k}, B_{m, k}, U_{m, k}, m_{u, 1}\right\}$, for $m \in[1, n-1]$ and $k \in[0, m]$, and their derivatives. Also, $\Omega_{0}^{\phi}:=0$. At this point we need to establish the maximum power of $\log R$ in the functions $\Omega_{n-1}^{\phi}$. Since we are collecting terms of order $R^{-n-1}$, naively we would say that a term collected this way could have a maximum power of $n+1$. Although, any good derivative or factor of $R^{-1}$ increases the order of the term without increasing the power of $\log R$. For instance, in the third term of equation (96) applied to $b$, the maximum power is $n$, because it has one good derivative and no factors of $R^{-1}$. With this in mind, we can split the functions $\Omega_{n-1}^{\phi}$ in the following way,

$$
\begin{aligned}
& \Omega_{n-1}^{g}=\sum_{p=0}^{n-1}(\log R)^{p} \Omega_{n-1, p}^{g}\left(\psi^{*}\right), \\
& \Omega_{n-1}^{b}=\sum_{p=0}^{n}(\log R)^{p} \Omega_{n-1, p}^{b}\left(\psi^{*}\right), \\
& \Omega_{n-1}^{u}=\sum_{p=0}^{n-1}(\log R)^{p} \Omega_{n-1, p}^{u}\left(\psi^{*}\right) .
\end{aligned}
$$

It is worth noting that the specific form of the $\Omega_{n-1, p}^{\phi}$ functions has no influence on the proof of our hypothesis, as long as it is possible to write (111). In fact (111) holds for a more general class of models than just (15), as will be discussed in the next section. Equation (111) allows us to integrate 110 in order to get the asymptotic behavior of $\mathcal{G}_{n}, \mathcal{B}_{n}$ and $\mathcal{U}_{n}$ in terms of $\left\{G_{m, k}, B_{m, k}, U_{m, k}, m_{u, 1}\right\}$. Let us begin with the first equation,

$$
R^{n} \nabla_{\psi}\left(\frac{1}{R^{n-1}} \nabla_{T} \mathcal{G}_{n}\right) \simeq \sum_{p=0}^{n-1}(\log R)^{p} \Omega_{n-1, p}^{g}\left(\psi^{*}\right)
$$

We make use of the following integral, $\forall q \neq 1$,

$$
\begin{aligned}
\int \frac{(\log R)^{p}}{R^{q}} d R & =-\frac{(\log R)^{p}}{(q-1) R^{q-1}}+\frac{p}{q-1} \int \frac{(\log R)^{p-1}}{R^{q}} d R \\
& =\sum_{i=0}^{p}-\frac{(\log R)^{i}}{(q-1)^{p-i+1} R^{q-1}} \frac{p !}{i !}
\end{aligned}
$$


to get,

$$
\begin{aligned}
\mathcal{G}_{n} \simeq & \sum_{p=0}^{n-1} \sum_{i=0}^{p}-\frac{(\log R)^{i}}{(n-1)^{p-i+1}} \frac{p !}{i !} \int_{T_{0}}^{T} \Omega_{n-1, p}^{g} d T^{\prime}+m_{g, n}\left(T_{0}\right) \\
= & \sum_{i=0}^{n-1}(\log R)^{i} \sum_{p=0}^{n}-\frac{1}{(n-1)^{p-i+1}} \frac{p !}{i !} \int_{T_{0}}^{T} \Omega_{n-1, p}^{g} d T^{\prime} \\
& +m_{g, n}\left(T_{0}\right) \\
= & \sum_{i=0}^{n-1}(\log R)^{i} G_{n, i}\left(\psi^{*}\right)
\end{aligned}
$$

for some scalar functions $G_{n, i}\left(\psi^{*}\right)$ and for all $n>1$. As in the flat case, we choose $\dot{g}_{n}=0$ for $n>1$, and the same applies for $b$ (for $n>1$ ) and $u$ (for $n>2$ ). In other words, if $g$ can be written as (105), then it can also be written as (106). Therefore, we have,

$$
g=\sum_{n=1}^{\infty} \sum_{k=0}^{n-1} \frac{(\log R)^{k} G_{n, k}\left(\psi^{*}\right)}{R^{n}}
$$

as desired. The $b$ equation likewise gives,

$$
\begin{aligned}
R^{n} \nabla_{\psi}\left(\frac{1}{R^{n-1}} \nabla_{T} \mathcal{B}_{n}\right) \simeq & -\nabla_{T} G_{n} \nabla_{T} G_{1,0} \\
& +\sum_{p=0}^{n}(\log R)^{p} \Omega_{n-1, p}^{b}\left(\psi^{*}\right)
\end{aligned}
$$

which we can integrate in order to get,

$$
\begin{aligned}
\mathcal{B}_{n} \simeq & \sum_{i=0}^{n}(\log R)^{i} \sum_{p=0}^{n}-\frac{1}{(n-1)^{p-i+1}} \frac{p !}{i !} \int_{T_{0}}^{T} \Omega_{n-1, p}^{b} d T^{\prime}+ \\
+ & \sum_{i=0}^{n-1}(\log R)^{i} \sum_{p=0}^{n-1}-\frac{1}{(n-1)^{p-i+1}} \frac{p !}{i !} \\
& \int_{T_{0}}^{T} \sum_{k=0}^{n-1} \nabla_{T} G_{n, k} \nabla_{T} G_{1,0} d T^{\prime}+m_{b, n}\left(T_{0}\right) \\
= & \sum_{i=0}^{n}(\log R)^{i} B_{n, i}\left(\psi^{*}\right)
\end{aligned}
$$

for all $n>1$. Thus, by induction, we get,

$$
b=\sum_{n=1}^{\infty} \sum_{k=0}^{n} \frac{(\log R)^{k} B_{n, k}\left(\psi^{*}\right)}{R^{n}} .
$$

Finally, the $u$ equation reads,

$$
R^{n-1} \nabla_{\psi}\left(\frac{1}{R^{n-2}} \nabla_{T} \mathcal{U}_{n}\right) \simeq \sum_{p=0}^{n-1}(\log R)^{p} \Omega_{n-1, p}^{u}\left(\psi^{*}\right)
$$

and integrating it along integral curves of $\psi^{a}$ and $\partial_{T}^{a}$ gives,

$$
\begin{aligned}
\mathcal{U}_{n} \simeq & \sum_{i=0}^{n-1}(\log R)^{i} \sum_{p=0}^{n-1}-\frac{1}{(n-2)^{p-i+1}} \frac{p !}{i !} \int_{T_{0}}^{T} \Omega_{n-1, p}^{U} d T^{\prime} \\
& +m_{u, n}\left(T_{0}\right) \\
= & \sum_{i=0}^{n-1}(\log R)^{i} U_{n, i}\left(\psi^{*}\right),
\end{aligned}
$$

for all $n>2$. By induction,

$$
u=\frac{m_{u, 1}}{R}+\sum_{n=2}^{\infty} \sum_{k=0}^{n-1} \frac{(\log R)^{k} U_{n, k}\left(\psi^{*}\right)}{R^{n}} .
$$

This concludes the proof. These results can be packaged in the following theorem.

Theorem 2. Let $X^{\underline{\alpha}}=\left(T, X^{\underline{i}}\right)$ be an asymptotically Cartesian coordinate system with an associated covariant derivative $\stackrel{\circ}{\nabla}$. The good-bad-ugly system defined as,

$$
\left\{\begin{array}{l}
\square \circ g=0 \\
\square \circ b=\left(\nabla_{T} g\right)^{2} \\
\square
\end{array},\right.
$$

where $\square^{\square}:=g^{a b} \stackrel{\circ}{\nabla}_{a} \stackrel{\circ}{\nabla}_{b}$ and $g$ is an asymptotically flat metric, admits a polyhomogeneous expansion near null infinity of the type,

$$
\begin{aligned}
& g=\sum_{n=1}^{\infty} \sum_{k=0}^{n-1} \frac{(\log R)^{k} G_{n, k}\left(\psi^{*}\right)}{R^{n}} \\
& b=\sum_{n=1}^{\infty} \sum_{k=0}^{n} \frac{(\log R)^{k} B_{n, k}\left(\psi^{*}\right)}{R^{n}} \\
& u=\frac{m_{u, 1}}{R}+\sum_{n=2}^{\infty} \sum_{k=0}^{n-1} \frac{(\log R)^{k} U_{n, k}\left(\psi^{*}\right)}{R^{n}},
\end{aligned}
$$

with initial data on $\mathcal{S}$ of the type,

$$
\left\{\begin{array}{l}
\left.g\right|_{\mathcal{S}}=\sum_{n=1}^{\infty} \frac{m_{g, n}}{R^{n}} \\
\left.b\right|_{\mathcal{S}}=\sum_{n=1}^{\infty} \frac{m_{b, n}}{R^{n}} \\
\left.u\right|_{\mathcal{S}}=\sum_{n=1}^{\infty} \frac{m_{u, n}}{R^{n}}
\end{array}\right.
$$

$$
\left\{\begin{array}{l}
\left.g\right|_{\mathcal{S}}=\sum_{n=1}^{\infty} \frac{m_{g, n}}{R^{n}} \\
\left.b\right|_{\mathcal{S}}=\sum_{n=1}^{\infty} \frac{m_{b, n}}{R^{n}} \\
\left.u\right|_{\mathcal{S}}=\sum_{n=1}^{\infty} \frac{m_{u, n}}{R^{n}}
\end{array} \quad,\left\{\begin{array}{l}
\left.\nabla_{T} g\right|_{\mathcal{S}}=O_{\mathcal{S}}\left(R^{-2}\right) \\
\left.\nabla_{T} b\right|_{\mathcal{S}}=O_{\mathcal{S}}\left(R^{-2}\right) \\
\left.\nabla_{T} u\right|_{\mathcal{S}}=O_{\mathcal{S}}\left(R^{-2}\right)
\end{array}\right.\right.
$$

where $m_{\phi, n}$ are scalar functions that are independent of $T$ and $R$. This is valid outside a compact ball centered at $R=0$.

Remark 3. As can be seen from (102), a sufficient condition to make the $\log R$ term in $U_{2}$ vanish, is that,

$$
\Delta m_{u, 1}=0
$$


which is exactly the same requirement as in the flat case. Although, with a general metric we cannot expect the $\log R$ terms to vanish at all orders, because order $n=3$ in $u$ is already coupled to the $b$ equation and might therefore inherit up to one power of $\log R$, depending on the form of the metric functions.

Remark 4. Once again one can see that the good-badugly system admits a static solution given by,

$$
\begin{aligned}
& R g=m_{g, 1}, \\
& R b=m_{b, 1}, \\
& R u=m_{u, 1},
\end{aligned}
$$

as long as the requirement $\not m_{\phi, 1}=0$ is fulfilled. If all further initial data are set to zero, then the series truncates at $n=1$ and all orders vanish except the first one. Note that this static solution differs from (92) because here the operator $\triangle$ is not necessarily the Laplace operator on the 2-sphere of radius $R$, but an analogous operator constructed from $g^{a b}$ that coincides with the former to leading order.

\section{STRATIFIED NULL FORMS}

We can generalize this proof to encompass models more complicated than the standard good-bad-ugly system. In fact there is a large class of terms that, added to the RHS of (15) require no significant changes in the induction proof. These terms are a generalization of the classical null forms, see 23, 24, that know about the different types of field. Let us define stratified null forms as terms that involve up to one derivative of the evolved fields and fall-off faster than $R^{-2}$ close to null infinity. For example, a term which has one good derivative, one bad derivative and no explicit dependence on coordinates, say,

$$
\nabla_{\underline{\psi}} g \nabla_{\psi} b
$$

is necessarily $o^{+}\left(R^{-2}\right)$, and is therefore a stratified null form. Another type of term that fulfills this requirement is one which is quadratic in bad derivatives, but has one power of $R^{-1}$, say,

$$
\frac{1}{R} \nabla_{\underline{\psi}} g \nabla_{\underline{\psi}} b .
$$

Finally, a term where any derivative hits an ugly field and a bad derivative hits any field, say,

$$
\nabla_{a} u \nabla_{\underline{\psi}} b
$$

is also a stratified null form, because any derivative hitting an ugly field, necessarily improves its decay. We will need this definition because it distinguishes the terms that significantly change our proof from those that do not. Let us replace our earlier system with

$$
\begin{aligned}
& \stackrel{\circ}{\square} g=N_{g}, \\
& \square \circ b=\left(\nabla_{T} g\right)^{2}+N_{b}, \\
& \square \circ u=\frac{2}{R} \stackrel{\circ}{\nabla}_{T} u+N_{u},
\end{aligned}
$$

where $N_{\phi}$ are arbitrary linear combinations of stratified null forms. As stratified null forms are at least of order $o^{+}\left(R^{-2}\right)$, regardless of any of these terms we add to the RHS of the good-bad-ugly system, the first order equations 102 remain the same, as they are the result of collecting terms proportional to $R^{-2}\left(R^{-3}\right.$ in the $u$ case),

$$
\begin{aligned}
& \nabla_{\psi} \nabla_{T} \mathcal{G}_{1} \simeq 0 \\
& 2 R \nabla_{\psi} \nabla_{T} \mathcal{B}_{1} \simeq-\left(\nabla_{T} G_{1}\right)^{2}, \\
& 2 R \nabla_{\psi} \nabla_{T} \mathcal{U}_{2} \simeq \tilde{\Delta} m_{u, 1} .
\end{aligned}
$$

Stratified null forms will, in general, contribute to the next order, however they will not contain derivatives of $\mathcal{G}_{2}, \mathcal{B}_{2}$ or $\mathcal{U}_{3}$. This is true for all $n$. At each step we collect terms of order $R^{-n-1}$ to find equations for $\mathcal{G}_{n}$, $\mathcal{B}_{n}$ or $\mathcal{U}_{n}$ and stratified null forms will only contain derivatives of $\left\{G_{m, k}, B_{m, k}, U_{m, k}, m_{u, 1}\right\}$, for $m \in[1, n-1]$ and $k \in[0, m]$. This implies that any terms arising from stratified null forms can be absorbed into $\Omega_{n-1}^{\phi}$ so that we get (cf. 110),

$$
\begin{aligned}
& R^{n} \nabla_{\psi}\left(\frac{1}{R^{n-1}} \nabla_{T} \mathcal{G}_{n}\right) \simeq \Omega_{n-1}^{\prime g}, \\
& R^{n} \nabla_{\psi}\left(\frac{1}{R^{n-1}} \nabla_{T} \mathcal{B}_{n}\right)+\nabla_{T} G_{n} \nabla_{T} G_{1} \simeq \Omega_{n-1}^{\prime b}, \\
& R^{n-1} \nabla_{\psi}\left(\frac{1}{R^{n-2}} \nabla_{T} \mathcal{U}_{n}\right) \simeq \Omega_{n-1}^{\prime u},
\end{aligned}
$$

where $\Omega_{n-1}^{\prime \phi}$ are just $\Omega_{n-1}^{\phi}$, as defined earlier, plus any extra terms coming from $N_{\phi}$. Naturally, (111) is still valid and hence $g, b$ and $u$ can be written as polyhomogeneous functions (113), (116) and (118). Although we can expect the final recursion relations of the $g, b$ and $u$ fields to change in general, the induction proof remains unchanged. Therefore, regardless of the addition of any stratified null forms to the good-bad-ugly system, we have the following result,

$$
\begin{aligned}
& g=\sum_{n=1}^{\infty} \sum_{k=0}^{n-1} \frac{(\log R)^{k} G_{n, k}\left(\psi^{*}\right)}{R^{n}} \\
& b=\sum_{n=1}^{\infty} \sum_{k=0}^{n} \frac{(\log R)^{k} B_{n, k}\left(\psi^{*}\right)}{R^{n}} \\
& u=\frac{m_{u, 1}}{R}+\sum_{n=2}^{\infty} \sum_{k=0}^{n-1} \frac{(\log R)^{k} U_{n, k}\left(\psi^{*}\right)}{R^{n}},
\end{aligned}
$$

This implies that we can generalize Theorem 2 in order to incorporate stratified null forms.

Theorem 3. Let $X^{\underline{\alpha}}=\left(T, X^{\underline{i}}\right)$ be an asymptotically Cartesian coordinate system with an associated covariant derivative $\stackrel{\circ}{\nabla}$. The good-bad-ugly system defined as,

$$
\begin{aligned}
& \stackrel{\circ}{\square} g=N_{g}, \\
& \stackrel{\circ}{\square} b=\left(\nabla_{T} g\right)^{2}+N_{b}, \\
& \stackrel{\circ}{\square} u=\frac{2}{R} \stackrel{\circ}{\nabla}_{T} u+N_{u},
\end{aligned}
$$


where $N_{\phi}$ are arbitrary linear combinations of stratified null forms, $\stackrel{\square}{\square}:=g^{a b} \stackrel{\circ}{\nabla}_{a} \stackrel{\circ}{\nabla}_{b}$ and $g_{a b}$ is an asymptotically flat metric, admits a polyhomogeneous expansion near null infinity of the type,

$$
\begin{aligned}
& g=\sum_{n=1}^{\infty} \sum_{k=0}^{n-1} \frac{(\log R)^{k} G_{n, k}\left(\psi^{*}\right)}{R^{n}} \\
& b=\sum_{n=1}^{\infty} \sum_{k=0}^{n} \frac{(\log R)^{k} B_{n, k}\left(\psi^{*}\right)}{R^{n}} \\
& u=\frac{m_{u, 1}}{R}+\sum_{n=2}^{\infty} \sum_{k=0}^{n-1} \frac{(\log R)^{k} U_{n, k}\left(\psi^{*}\right)}{R^{n}},
\end{aligned}
$$

with initial data on $\mathcal{S}$ of the type,

$$
\left\{\begin{array}{l}
\left.g\right|_{\mathcal{S}}=\sum_{n=1}^{\infty} \frac{m_{g, n}}{R^{n}} \\
\left.b\right|_{\mathcal{S}}=\sum_{n=1}^{\infty} \frac{m_{b, n}}{R^{n}} \\
\left.u\right|_{\mathcal{S}}=\sum_{n=1}^{\infty} \frac{m_{u, n}}{R^{n}}
\end{array} \quad,\left\{\begin{array}{l}
\left.\nabla_{T} g\right|_{\mathcal{S}}=O_{\mathcal{S}}\left(R^{-2}\right) \\
\left.\nabla_{T} b\right|_{\mathcal{S}}=O_{\mathcal{S}}\left(R^{-2}\right) \\
\left.\nabla_{T} u\right|_{\mathcal{S}}=O_{\mathcal{S}}\left(R^{-2}\right)
\end{array}\right.\right.
$$

where $m_{\phi, n}$ are scalar functions that are independent of $T$ and $R$. This is valid outside a compact ball centered at $R=0$.

\section{CONCLUSIONS}

In this paper we laid out a heuristic method to predict the decay of terms beyond first order in $R^{-1}$ in the good-bad-ugly system. In its most general form the model consists of a set of coupled nonlinear wave equations in which the three different classes of fields have different asymptotic properties near null-infinity. We began with the simplest form of this system, as introduced in 19, built from the Minkowski metric and found that near null infinity there exist formal solutions to this model in which the bad field may have $\log R$ terms at every order in $R^{-1}$, the ugly field may have logs from second order onward, whereas the good field has no logs at all. We showed furthermore a recursion relation that allows us to find each order in $R^{-1}$ from the previous one to arbitrary order. The method is, however, heuristic because we have not shown that all physically relevant solutions of the good-bad-ugly system admit expansions of this form.

This was used as a warm-up for a more general system built from a general asymptotically flat metric. Keeping the metric functions fairly general, insisting essentially only that they be analytic functions of the evolved fields, we showed by induction that there is a class of asymptotic solutions near null infinity characterized by polyhomogeneous functions, the main difference between the three types of fields being the order at which log terms are first allowed to appear. As the metric components were intentionally left free, a full recursion relation for a general metric was not possible. However, we anticipate no reason why this method would not be straightforwardly applicable to any metric with these requirements in order to find such relations. In a final generalization to the model we considered the effect of non-linearities of a special class that we call stratified null forms. By definition these are precisely the terms involving up to one derivative of the evolved fields that fall-off faster than $O\left(R^{-2}\right)$. All of our results are subsumed within Theorem 3 which says that the same type of expansion also works out in the presence of arbitrary stratified null forms.

The restriction of having just one field of each type in our model is purely for simplicity. A more general setup with sets of fields of each type just requires more book-keeping. In fact, in future work, we aim to apply this method to the EFE in GHG to predict that its asymptotic solutions can be written as polyhomogeneous functions near null infinity. Due to the complexity of the full field equations, the asymptotic system will presumably be very long, but we anticipate that the the non-linearities studied in the good-bad-ugly system already capture the subtleties of those in GR. By finding the first few orders of a polyhomogeneous expansion of asymptotic solutions to the EFE, we expect to be able to recover the peeling properties, or a polyhomogeneous generalization, of the gravitational field.

Similar polyhomogeneous behavior of the gravitational field close to spatial and null infinity has been obtained by means of the conformal Einstein field equations in [25, 26] - see also 27 30 for further discussion of peeling. The polyhomogeneous expansions described in 25, 26] are formal in the sense that the appropriate energy estimates needed to rigorously prove that these expansions arise as an actual solution from some given initial data are still lacking. The polyhomogeneity result we potentially expect to obtain by exploiting the methods presented above for the EFE in GHG would be formal in the same sense. Ultimately we aim to make contact with the expansions given in 31. in harmonic gauge, in which no log terms are present. It is worth mentioning that the logarithmic terms appearing in the expansions described in [25, 26] have a very different origin from those analyzed here. In the case of the good-bad-ugly model and the EFE in GHG the logarithmic terms appear in the asymptotic expansion due to the form of the non-linearities in the equations, while the logarithmic terms of [25, 26] appear even in a linear context, such as the spin-2 field equations, in a Minkowski background in the framework of the cylinder at spatial infinity as discussed in 32 .

We furthermore hope that this work will be a stepping stone towards a full regularization of GR in GHG at null-infinity. Knowing from the outset where the logs may appear up to arbitrary order, one can employ a 'subtract-the-logs' strategy as the one used in [19] in order to treat these divergent terms, or indeed attempt to carefully choose gauge source functions that eradicate them all together. 


\section{ACKNOWLEDGMENTS}

The Authors wish to thank Alex Vañó-Viñuales for helpful comments on the manuscript. MD acknowledges support from FCT (Portugal) program $\mathrm{PD} / \mathrm{BD} / 135511 / 2018$, DH acknowledges support from the FCT (Portugal) IF Program IF $/ 00577 / 2015$, PTDC/MAT- APL/30043/2017. JF acknowledges support from FCT (Portugal) programs PTDC/MAT-
APL/30043/2017, UIDB/00099/2020. EG gratefully acknowledges support from the European Union's H2020 ERC Consolidator Grant "Matter and Strong-Field Gravity: New Frontiers in Einstein's Theory," Grant Agreement No. MaGRaTh-646597. EG also acknowledges support from the European Union (through the PO FEDER-FSE Bourgogne 2014/2020 program) and the EIPHI Graduate School (contract ANR-17-EURE0002) as part of the ISA 2019 project.
[1] R. Penrose, Phys. Rev. Lett. 10, 66 (1963).

[2] E. Newman and R. Penrose, Journal of Mathematical Physics 3, $566 \quad$ (1962), https://doi.org/10.1063/1.1724257.

[3] H. Bondi, M. G. J. van der Burg, and A. W. K. Metzner, Proceedings of the Royal Society of London. Series A, Mathematical and Physical Sciences 269, 21 (1962)

[4] R. Sachs, Phys. Rev. 128, 2851 (1962)

[5] R. Sachs, Proc. Roy. Soc. Lond. A 270, 103 (1962).

[6] P. Hübner, Class. Quantum Grav. 16, 2823 (1999).

[7] P. Hübner, Class. Quantum Grav. 18, 1871 (2001).

[8] G. Doulis and J. Frauendiener, (2016), arXiv:1609.03584 [gr-qc].

[9] H. Friedrich, Proc. Roy. Soc. London A 375, 169 (1981).

[10] H. Friedrich, Proc. Roy. Soc. London A 378, 401 (1981).

[11] J. Winicour, Living Rev. Relativity 15, 2 (2012), [Online article].

[12] A. Zenginoglu, A conformal approach to numerical calculations of asymptotically flat spacetimes, Ph.D. thesis, Potsdam U., Inst. of Math. (2007), arXiv:0711.0873 [grqc].

[13] A. Vañó-Viñuales, S. Husa, and D. Hilditch, Class. Quant. Grav. 32, 175010 (2015), arXiv:1412.3827 [gr-qc]

[14] A. Vañó-Viñuales and S. Husa, Proceedings, Spanish Relativity Meeting: Almost 100 years after Einstein Revolution (ERE 2014), J. Phys. Conf. Ser. 600, 012061 (2015), arXiv:1412.4801 [gr-qc]

[15] A. Vañó-Viñuales, Free evolution of the hyperboloidal initial value problem in spherical symmetry, Ph.D. thesis, $\mathrm{U}$. Iles Balears, Palma (2015), arXiv:1512.00776 [gr-qc].

[16] D. Hilditch, (2015), arXiv:1509.02071 [gr-qc]]

[17] D. Hilditch, E. Harms, M. Bugner, H. Rüter, and B. Brügmann, Class. Quant. Grav. 35, 055003 (2018)
arXiv:1609.08949 [gr-qc]

[18] E. Gasperin and D. Hilditch, Class. Quant. Grav. 36, 195016 (2019), arXiv:1812.06550 [gr-qc].

[19] E. Gasperin, S. Gautam, D. Hilditch, and A. VañóViñuales, (2019), arXiv:1909.11749 [gr-qc]

[20] S. Gautam, A. Vañó Viñuales, D. Hilditch, and S. Bose, (2021), arXiv:2101.05038 [gr-qc].

[21] H. Lindblad and I. Rodnianski, Comptes Rendus Mathematique 336, 901 (2003).

[22] J. Keir, ArXiv e-prints (2018), arXiv:1808.09982 [math.AP]

[23] S. Klainerman, Communications on Pure and Applied Mathematics 33, 43 (1980)

[24] C. Sogge, Lectures on nonlinear wave equations, Monographs in analysis No. Bd. 2 (International Press, 1995).

[25] E. Gasperín and J. A. Valiente Kroon, Classical and Quantum Gravity 34, 195007 (2017), arXiv:1706.04227 [gr-qc]

[26] H. Friedrich, Journal of Geometry and Physics 24, 83 (1998).

[27] P. T. Chruściel, M. A. H. MacCallum, and D. B. Singleton, Phil. Trans. Roy. Soc. Lond. A 350, 113 (1995).

[28] S. Novak and J. N. Goldberg, Gen. Relativ. Gravit. 14, 655 (1982).

[29] J. Winicour, Found. Phys. 15, 605 (1985).

[30] S. Klainerman and F. Nicoló, The Evolution Problem in General Relativity (Birkhäuser Boston, 2003).

[31] G. Satishchandran and R. M. Wald, Phys. Rev. D 99, 084007 (2019), arXiv:1901.05942 [gr-qc]

[32] J. A. Valiente Kroon, in The Conformal Structure of Spacetimes: Geometry, Numerics, Analysis, Lecture Notes in Physics, edited by J. Frauendiener and H. Friedrich (Springer, 2002) p. 135. 